\title{
B-mode Real Time Ultrasonographic imaging of the Heart in Sheep
}

\author{
Aissi. A \\ Surgery and Veterinary Imaging Service, Veterinary Department, \\ Veterinary sciences Institute, Batna University, \\ Batna 05000, Algeria. \\ E-mail: chirurgie05@yahoo.fr \\ Received: 08-06-2011, Accepted: 16-06-2011, Published Online: 05-09-2011 \\ doi: $10.5455 /$ vetworld.2011.467-469
}

\begin{abstract}
The anatomical structure of normal heart was studied in vitro through ultrasonography of 27 healthy hearts of sheep collected following slaughter. Ultrasonography was done in the water with $25 \mathrm{o} C$ by sectorial transducer from $2 \mathrm{~cm}$ distance. In the laboratory, the waves of ultrasonography were sent from right ventricle (RV). The thickness of left ventricle wall (LV) $(20.69 \pm 2.628 \mathrm{~mm})$, right ventricle wall $(\mathrm{RV})(8.10 \pm 2.138 \mathrm{~mm})$ and inter ventricular space (IVS) $(18.925 \pm 1.984 \mathrm{~mm})$ were measured by ultrasonography. After preparing ultrasonogrphes obtained by in vitro ultrasonography were compared with natural anatomical views. Also biometrical measurements were compared with sizes were measured by ultrasonography measurement. Biometrical measurements were performed by a special ruler (caliper) right ventricle thickness $(7.6 \pm 1.957 \mathrm{~mm})$, left ventricle thickness $(19.20 \pm 1.563 \mathrm{~mm})$ and inter-ventricular space thickness is $(17.981 \pm 1.786 \mathrm{~mm})$.
\end{abstract}

Key worlds: Ultrasonography, heart, sheep, anatomy.

\section{I ntroduction}

Twenty years ago, ultrasonography was rarely used in animal practice. At that time it was only available in some tertiary referral centers and the equipment was well below the currently accepted standards in terms of image resolution and processor power (Allen,1982). Today, echocardiography is performed as the 'in clinic' diagnostic gold standard for most cardiac conditions. Lower equipment costs, increased under-graduate and post-graduate training opportunities, and improved image quality have made this imaging modality accessible to many small animal practitioners (Allen; 1982, Fortuin et al., 1972).

Echocardiography is the accepted term for the study of cardiac ultrasound. Although a relatively new tool for the study of the heart in man it has already found wide acceptance in the area of cardiac research and in the study of clinical cardiac disease. Animals had often been used in the early experiments with cardiac ultrasound, but only recently has echocardiography been used as a research and clinical tool in veterinary medicine. In this report echocardiography is used in the research of anesthetic effects on ventricular function and clinically in the diagnosis of congestive cardiomyopathy in a cat, ventricular septal defect in a calf, and pericardial effusion in a dog. Echocardiography is now an important adjunct to the field of veterinary cardiology (Pipers et al., 1978).

Ultrasonography can be used for recognizing anatomical and structural disorders in the interior of the body organs in the clinic. A sonologist must be familiar with the normal ultrasonographic appearance (echotexture) of an organ to differentiate it from the abnormal views (Anderson, 1992; Braun and Gotz, 1994; Braun and Sicher, 2006). The heart is an important body organ. However, its normal anatomical structure can not be studied without dissection and surgery. In the present study, the anatomical structure of normal heart was studied in vitro by using ultrasonography of 27 healthy hearts of sheep after slaughter.

\section{Materials and Methods}

For ultrasonographic study of the heart anatomy in vitro, 27 healthy sheep heart were collected from Batna slaughterhouse for prepare ultrsonographe from the heart in laboratory. The hearts were transferred to surgery and veterinary imaging service, veterinary sciences institut, Batna University. Ultrasonography was done in the water with $25{ }^{\circ} \mathrm{C}$ by sectorial transducer (Mylab 40 XVision Biosound Esaote), 
from $2 \mathrm{~cm}$ distance (Fig 1). In the laboratory, the waves of ultrasonography were sent from right ventricle (RV). The thickness of left ventricle wall (LV), right ventricle wall (RV) and inter ventricular space (IVS) were measured by ultrasonography (Fig 2). After preparing ultrasonogrphes obtained by in vitro ultrasonography were compared with natural anatomical views. Also biometrical measurements were compared with sizes were measured by ultrasonography measurement. Biometrical measurements were performed by a special ruler(caliper).

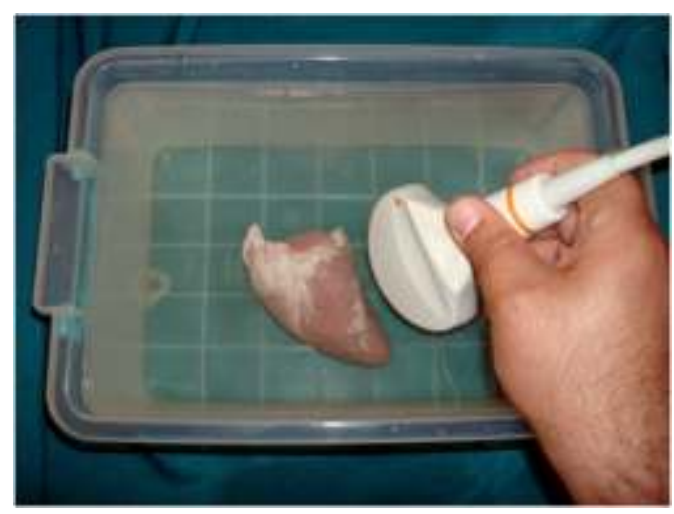

Figure-1. sheep heart Ultrasonography explore by sectorial tranducer probe.

\section{Results}

Thickness of right ventricle (RV), thickness of left ventricle wall (LV), and thickness of inter ventricular space (IVS) was observed in figure- 2 .

The ultrasonography measurements of right ventricle wall thickness and left ventricle wall

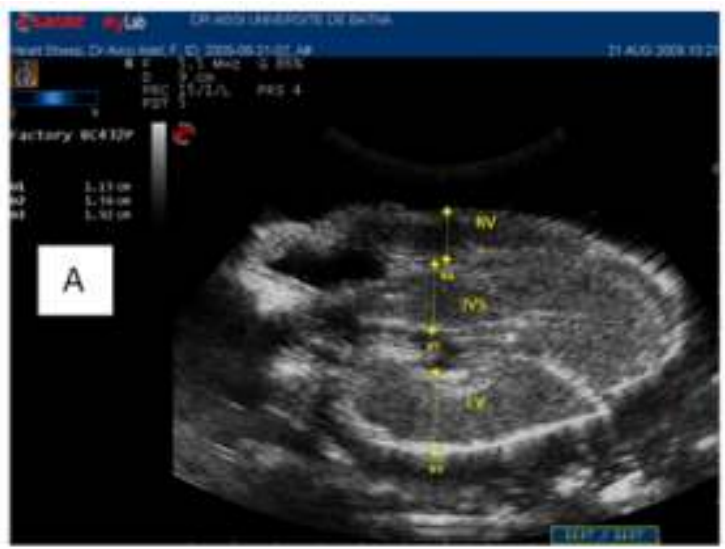

thickness and inter-ventricular space thickness were written in the Table-1. After the hearts were sonographied by in vitro, they were cut by a scalpel and were measured by a special ruler (caliper). The results of this part were written in Table 2 . In figure-3 was shown the anatomical measuring of the heart.

\section{Discussion}

Ultrasonography is relatively a new technique. However, these days this technique is routinely used for pregnancy diagnosis in small and large animal and for the detection of lesions in various body organs (Aissi et al 2008(a), Aissi et al., 2008 (b)). Therefore, enough information is available in the literature about normal and abnormal echotexture of various body organs (Dyce et al., 1996; Braun and Sicher, 2006). Scientists have used ultrasonography for the study of superficial lymph nodes, spleen, kidneys, urinary bladder, ovaries, uterus, testes, liver, gall bladder, and also mucosa of reticulum, omasum, abomasums and rumen. Furthermore, rumen, omasum and abomausm have physiological diagnostic differences with other organs. Inflammation, adhesions, cystic lesions, abscesses and other lesions can easily be diagnosed by ultrasonography (Ahmad et al., 1991; Jackson and Salter, 1997; Nautrup, 2000).

Importance of the heart and its location in the chest area indicates that the ultrasonography could be a better technique for the diagnosis of heart disorders. A comparison of results obtained by ultrasonographic and biometrical measurement methods shows that the results of both the methods were quite comparable. It indicates that in vivo ultrasonography can be used for the anatomical and biometrical study of heart. The values for the thickness of left ventricle wall, right

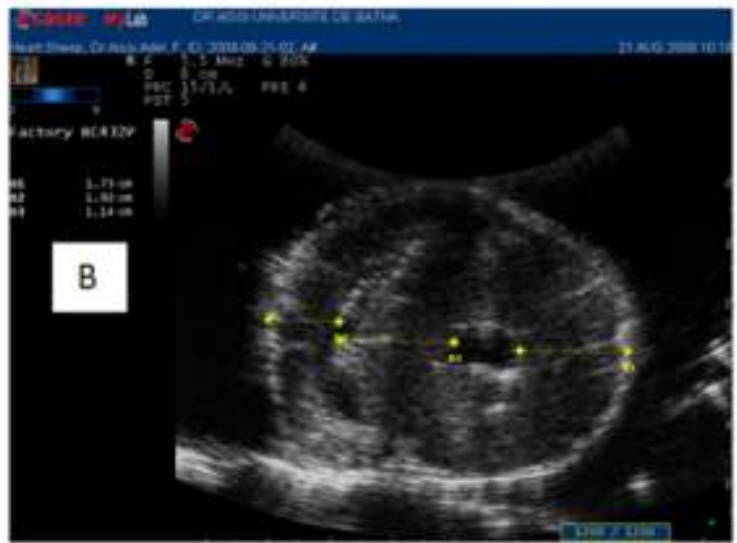

Figure-2. Ultrasonographes from right ventricle (RV), left ventricle (LV), and inter ventricular Wall thickness (IVS). A : Longitudinal section B:Transverse section 
Table- 1 results of measuring sheep hearts ventricle walls by Ultrasonography ( $\mathrm{mm}$ )

\begin{tabular}{ccc}
\hline Left ventricle wall thickness & Right ventricle wall thickness & Inter-ventricular space thickness \\
\hline $20.69 \pm 2.628$ & $8.10 \pm 2.138$ & $18.925 \pm 1.984$ \\
\hline
\end{tabular}

Table- 2 results of sheep heart ventricle walls and inter ventricular wall thickness at anatomical way with a ruler $(\mathrm{mm})$.

\begin{tabular}{ccc}
\hline Left ventricle diameter & Right ventricle diameter & Inter-ventricular space diameter \\
\hline $19.20 \pm 1.563$ & $7.6 \pm 1.957$ & $17.981 \pm 1.786$ \\
\hline
\end{tabular}

ventricle wall and inter-ventricular space can be used as basis for detecting changes in these parts due to any heart disease (karimi et al., 2008).

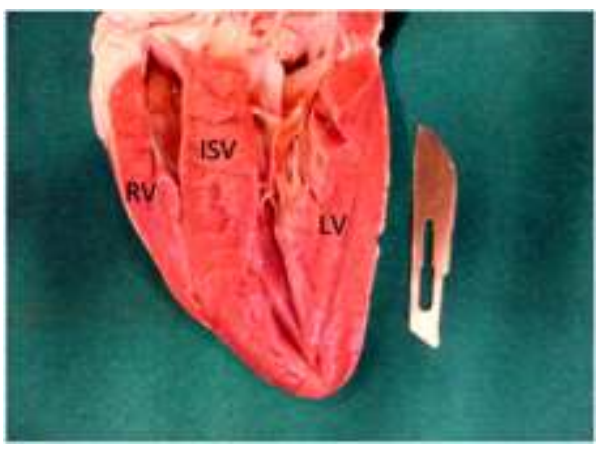

Figure - 3. Anatomical measurement: RV wall, LV wall and IVS in anatomical way. RV (right ventricle), IVS (inter-ventricular space), LV (left ventricle).

\section{Acknowledgement}

Author is highly thankful to Mr Boukejouta, M. and Hospimed Afric For materials and Ultrasonography machine support.

\section{References}

1. Ahmad, N., D. E. Noakes and A. L. Subandrio, (1991). B- mode real time ultrasonographic imaging of testis and epididymis of sheep and goats. Vet. Rec., 128: 491-496.

2. Anderson, N. V., (1992). Veterinary Gastroenterology. 2nd Ed., Lea and Febiger, Philadelphia, USA.

3. Allen D. G (1982).Echocardiography as a Research and Clinical Tool in Veterinary Medicine. Can Vet J. 23(11): 313-316.

4. Aissi.A, Slimani.C.(2009). Ultrasound Diagnosis of Cholecystitis in a Dog. Global Veterinaria 3 (6): 514-515.

5. Aissi.A, (2008). Ultrasonographic Diagnosis of Transitional cell Carcinoma of the urinary bladder, Online $J$ Vet Res, 12 (2) 67-69

6. Braun, U. and D. Sicher, (2006). Ulrasonography of the spleen in 50 healthy cows. Vet. J., 171(3): 513-518.

7. Braun, U. and M. Gotz, (1994). Ultrasonography of the reticulum in cows. Amer. J. Vet. Res., 55(3): 325-332.

8. Dyce, K. M., W. O. Sank and C. J. G. Wensing, (1996). Textbook of Veterinary Anatomy. 2nd Ed., W. B. Saunders Company, Philadelphia, USA.

9. Fortuin NJ, Hood WP, Jr, Craige E. (1972). Evaluation of left ventricular function by echocardiography. Circulation. 46(1):26-35.

10. Nautrup, C. P., (2000). An Atlas and Textbook of Diagnostic Ultrasonography of the Dog and Cat. Manson Publishing Ltd., Hannover Germany, pp: 1-5.

11. Karimi H. (2008). in vitro ultrasonography of the normal sheep heart.Pak. Vet. J., 28(2): 92-94.

12. Jackson, P. and J. Salter, (1997). Cardiovascular diseases in cattle. In Practice, 19(9): 475-476.

13. Pipers FS, Rings DM, Hull BL, Hoffsis GF, Reef V, Hamlin RL.(1978). Echocardiographic diagnosis of endocarditis in a bull. JAm Vet MedAssoc. Jun 1;172(11):1313-1316.

$* * * * * * * *$ 